\title{
Orbital polarization, surface enhancement and quantum confinement in nanocluster magnetism
}

\author{
Xiangang Wan, ${ }^{1}$ Lei Zhou, ${ }^{2}$ Jinming Dong, ${ }^{1}$ T. K. Lee, ${ }^{3}$ and Ding-sheng Wang ${ }^{4,5}$ \\ ${ }^{1}$ National Laboratory of Solid State Microstructures and Department of Physics, Nanjing University, Nanjing 210093, China \\ ${ }^{2}$ Department of Physics, The Hong Kong University of Science and Technology, Clear Water Bay, Kowloon, Hong Kong, China \\ ${ }^{3}$ Institute of Physics, Academia Sinica, Nankang, Taipei, Taiwan 11529 \\ ${ }^{4}$ Institute of Physics, Academia Sinica, Beijing 100080, China \\ ${ }^{5}$ Interdisciplinary Center of Theoretical Studies, Academia Sinica, Beijing 100080, China
}

(Received 22 December 2003; revised manuscript received 17 March 2004; published 18 May 2004)

\begin{abstract}
Within a rather general tight-binding framework, we studied the magnetic properties of $\mathrm{Ni}_{n}$ clusters with $n=9-60$. In addition to usual hopping, exchange, and spin-orbit coupling terms, our Hamiltonian also included orbital correlation and valence orbital shift of surface atoms. We show that orbital moment not only contributes appreciably to the total moment in this range of cluster size, but also dominates the oscillation of total moment with respect to the cluster size. Surface enhancement is found to occur not only for spin but, even stronger, also for orbital moment. The magnitude of this enhancement depends mainly on the coordination deficit of surface atoms, well described by a simple interpolation. For very small clusters $(n \leqslant 20)$, quantum confinement of $4 s$ electrons has drastic effects on $3 d$ electron occupation, and thus greatly influences both spin and orbital magnetic moments. With physically reasonable parameters to account for orbital correlation and surface valence orbital shift, our results are in good quantitative agreement with available experiments, evidencing the construction of a unified theoretical framework for nanocluster magnetism.
\end{abstract}

DOI: 10.1103/PhysRevB.69.174414

PACS number(s): 75.50.Tt, 36.40.Cg, 75.75.+a

\section{INTRODUCTION}

Theoretical and experimental studies have demonstrated that magnetism of materials is strongly affected by their dimensionalities and sizes. This has been clearly illustrated in multilayers and gas-phase clusters whose sizes can be reduced to atomic level. The strong size dependence of magnetic moment opens new possibilities to design materials with specific and tailored properties. Recently, transition metal nanoclusters have attracted considerable attention due to both theoretical and practical interests. Using the SternGerlach deflection technique, Billas et al. ${ }^{1,2}$ measured the magnetic moments of $\mathrm{Ni}, \mathrm{Fe}$, and $\mathrm{Co}$ clusters ranging from about 25 to 700 atoms. It was shown that small clusters usually possessed large magnetic moments, e.g., about $1 \mu_{B} /$ atom for $\mathrm{Ni}_{20-30}$. Using the same technique, Apsel et $a .^{3}{ }^{3}$ performed more accurate measurements for nickel clusters containing 5 to 740 atoms. In addition to an overall decrease with increasing cluster size, they found the magnetic moment to exhibit characteristic oscillations. A pronounced sharp minimum was found at $n=13$, and other less pronounced minima at $n=6,34$, and 56 (see dashed lines in Fig. 1). Different from Billias et al., Apsel et al. found that the approach to bulk magnetism was much slower. For example, the magnetic moment was larger than $0.70 \mu_{B}$ /atom even up to $n=500$. Very recently, Knickelbein ${ }^{4}$ again measured the magnetic moments of small Ni clusters containing 7-25 atoms. While his results confirmed the pronounced sharp moment minimum at $\mathrm{Ni}_{13}$ (Fig. 1), the measured moment values were about $0.2 \mu_{B}$ /atom smaller than those of Apsel et al. for most clusters. In general, all these experiments showed similar size dependences of magnetic moment, despite of some quantitative differences which may come from either experimental errors or the differences in the structure of the clusters.

In a theoretical discussion, Billias et al. ${ }^{2}$ proposed a simple magnetic shell model to account for the size dependences of magnetic moments for $\mathrm{Fe}$, $\mathrm{Co}$, and $\mathrm{Ni}$ clusters. They assumed their clusters to be structureless and consist of several spherical atomic shells. The magnetic moment of an atom on each shell depends only on its distance to the cluster surface. While this simple model yielded a correct decreasing trend of magnetic moment versus size, it failed to reproduce the oscillations superimposed on the overall decrease. It is well known that giant moments exist on surface atoms, as revealed in early 1980 s by either local spin density-

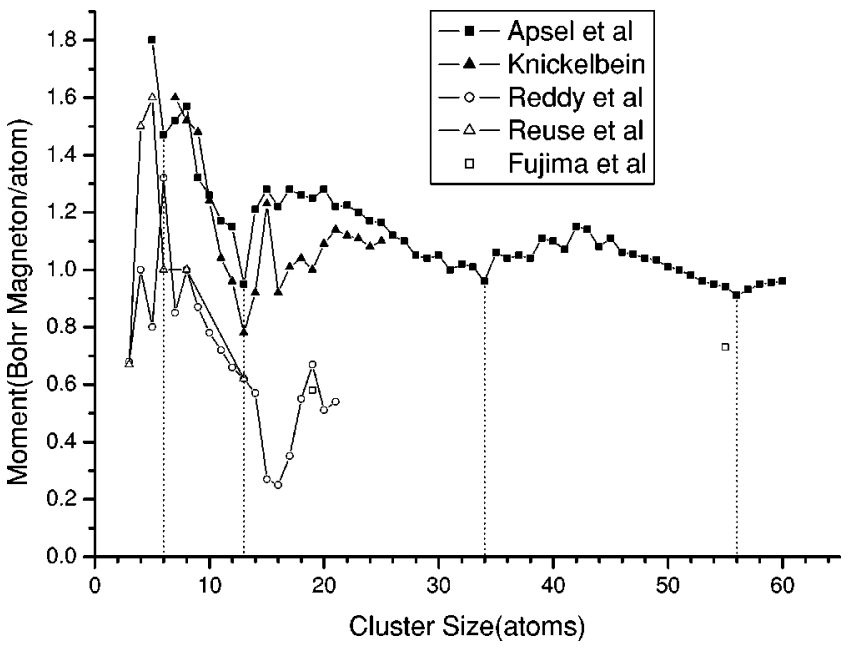

FIG. 1. Magnetic moments as a functions of cluster size of two experiments (solid symbols), compared with first-principles theoretical values (open symbols). Four dashed lines show the experimental moment minima positions. 
TABLE I. Surface enhancement of spin moment and core-level $\left(2 p_{3 / 2}\right)$ shift of Ni films as calculated from standard FLAPW method.

\begin{tabular}{lccc}
\hline \hline System & $Z$ & $\mu\left(\mu_{B}\right)$ & Shift $(\mathrm{eV})$ \\
\hline Bulk & 12 & 0.561 & \\
(111) film center & 12 & 0.613 & \\
(100) film center & 12 & 0.619 & \\
(111) film surface & 9 & 0.625 & 0.291 \\
(100) film surface & 8 & 0.675 & 0.354 \\
(111) monolayer & 6 & 0.892 & \\
(100) monolayer & 4 & 1.014 & \\
\hline \hline
\end{tabular}

functional (LSDA) calculations and later substantiated by experiments (see, for example, a review by Freeman and $\mathrm{Wu}^{5}$ ). Table I lists some typical LSDA results for $\mathrm{Ni}$ systems in bulk and film geometries from standard full potential linearized augmented plan wave (FLAPW) calculations. ${ }^{6}$ For Ni systems, the spin moment per atom drops from $2 \mu_{B}$ for an isolated atom (Hund's rule), to $1.014 \mu_{B}$ for an isolated (100) monolayer (the coordination number $Z=4$ ), and $0.675 \mu_{B}$ for a surface atom of (100) five-layer slab $(Z=8)$, and finally to $0.561 \mu_{B}$ for a bulk crystal. Taking such $Z$ dependence into account, Jensen and Bennenmann ${ }^{7}$ proposed a coordination model for cluster magnetism, in which the magnetic moment of an atom was assumed to depend solely on its number of nearest neighbors $Z$ as in the film case. In the following sections, this idea will be tested thoroughly. We found that, despite of the complicated local environments for atoms on a cluster surface, this model still approximately holds for both spin and orbital moments in cluster magnetism.

On the other hand, Fujima and Yamaguchi ${ }^{8}$ considered the effects of volume confinement for delocalized atomic $s$ electrons in clusters. Strong bonding, less strong bonding, or anti-bonding states, depending on the global symmetry (denoted as capital $S, P, D, \ldots$ ) of the linear combination coefficients in the tight-binding (TB) terminology, were formed by the delocalized atomic $s$ orbits. This volume confinement raises the energy separations between shells with global $S, P$, $D$. . . symmetry. Upon changing the cluster size, whenever one of these global shells goes through the Fermi level, which locates near the top of denser $d$ bands, the total number of $s$ electrons changes abruptly, which in turn, changes the number of holes in the $d$ bands. This effect offers a possible explanation for the oscillatory size dependence of magnetic moment, but Fujima and Yamaguchi provided only a qualitative suggestion. In order to convincingly identify the oscillations observed in experiments to this quantum confinement effect, a more rigorous treatment of the $s$ - $d$ coupling and cluster structures should be made.

Ideally, one expects that an $a b$ initio calculation (for example, in the LSDA framework) could automatically take the above-mentioned surface and quantum confinement effect into account, since it can precisely determine the charge of electronic density due to the spillover on the surface, and the redistributions of the $s-d$ electrons. But calculated results reported by Reuse et al. ${ }^{9}$ (open triangles in Fig. 1) and Reddy et al. $^{10}$ (open circles in Fig. 1) were substantially smaller by about $0.3-0.6 \mu_{B} /$ atom. And they failed to reproduce the moment variations-even the most pronounced sharp minimum at $\mathrm{Ni}_{13}$. Using spin-polarized discrete variational method, Fujima and Yamaguchi also studied the magnetic properties of $\mathrm{Ni}$ clusters. ${ }^{11}$ The calculated magnetic moment was $0.58 \mu_{B}$ /atom for $\mathrm{Ni}_{19}$, agreeing well with Reddy et al. and $0.73 \mu_{B}$ /atom for $\mathrm{Ni}_{55}$. In general, all calculations based upon first principles showed poor agreements by underestimating substantially the spin moment (Fig. 1). Considering the successes of LSDA in bulk and surface magnetism, where the calculated moments of $3 d$ metals and alloys are usually in agreement with experiments within about $0.05 \mu_{B}$ /atom, the failure in clusters is more or less out of initial expectation.

The TB method has been used to deal with even larger clusters. The results are, in general, similar to the firstprinciples ones. Using a model Hamiltonian which took into account the electron spillover at cluster's surfaces, Weissmann and co-workers ${ }^{12}$ studied the size dependences of magnetic moments for fcc and bcc clusters up to 177 atoms. However, similar to the first-principles results, their calculated moments were again mostly smaller than the experimental ones. Alonso and co-workers also made a thorough study for Ni clusters. ${ }^{13-15}$ Using a special parametrization of the orbital energies, they could get moment values much higher than the LSDA results, and brought, especially in the small size range, the moment values closer to the experimental one. However, their results are far from satisfactory, and the agreement seems somewhat an artifact in their parametrization scheme (see a discussion in Sec. IV A).

We note that all those previous calculations mentioned above did not consider the orbital magnetic moment. Through a general argument, it was shown that orbital correlation has stronger effects in low-dimensional transition metal systems than in bulk crystals by leading to orbital polarized ground states. ${ }^{16}$ This was demonstrated also by firstprinciples calculations for isolated or substrate supported linear-chain systems, ${ }^{17}$ and adatoms on metallic substrates. ${ }^{18}$ Presumably, orbital interactions should be much more sensitive to the details of the local structures. Therefore, we expect that the environment dependence of orbital moment, if not fully quenched, should be even more remarkable than that of spin moment. Very recently, using a TB scheme which includes orbital correlation and spin-orbit interactions, Guirado-López et al. ${ }^{19}$ studied the magnetic moments for $\mathrm{Ni}$ clusters. Their results confirmed the importance of orbital moments in Ni clusters. Since they neglected the lowsymmetry clusters and considered only a few fcc or icosahedral geometries, their paper did not show a rather complete size dependence of the moment to compare with experiments. It is thus desirable to investigate the magnetic moments for an as complete as possible series of Ni clusters, with orbital correlation included, to check whether the disagreement between experiments and theoretical works is due to the neglect of orbital contribution.

The present paper is organized in the following way. The geometrical structures used will be discussed in Sec. II, and a detailed description of our model Hamiltonian will be given in Sec. III. Calculation results are discussed in Sec. IV, in 
which the importance of orbital contribution is highlighted. We analyze the surface enhancement effect in Sec. V, and the quantum confinement effect in Sec. VI. Finally, Sec. VII will summarize the results of present study.

\section{CLUSTER GEOMETRY}

Itinerant electron magnetism is sensitive to the atomic structure of the system. The main factors to influence the magnetic properties of small Ni clusters are (i) low atomic coordination and the relaxation of interatomic distances for surface atoms; (ii) quantum confinement of delocalized $s$ electrons which controls indirectly the number of holes in the $d$ bands. The first factor is basically a local geometrical effect, and the latter one is global. Both factors may play important roles in determining the electronic structure. A good geometrical structure is therefore a crucial start in any attempt to interpret the nanocluster magnetism. Experimental determination of a cluster geometry is difficult, since most clusters are too large for spectroscopic probes but too small for diffraction probes. Therefore, people usually employ theory to determine the cluster structures and subsequently compare some calculated properties with experiments. Unfortunately, the structures of most clusters (say, over a few tens of atoms) have not been accurately determined by $a b$ initio calculations because of the enormous computational works involved. Instead, geometrical structures of nanoclusters are available mostly from calculations using semiempirical interatomic potential.

Geometries of $\mathrm{Ni}_{n}$ with $n \leqslant 20$ were obtained by molecular-dynamics simulations with a semiempirical manybody potential, ${ }^{20,21}$ and it is found that for small Ni clusters the structure is icosahedral. ${ }^{14}$ This structure has also been verified subsequently by Montejano-Carrizales, Iñiguez, Alonso, and López (MIAL), Ref. 22 using an embeddedatom method. For $n$ larger than 13, two types of icosahedral growth were proposed, usually called as Mackey icosahedra (MIC) and polyicosahedra (TIC) ${ }^{13,22}$ According to the MIAL paper, the TIC growth is favored for $14 \leqslant n \leqslant 27$ and $57 \leqslant n$ $\leqslant 67$, but the MIC growth is favored for $28 \leqslant n \leqslant 56$. This evolution of structural symmetry is shown in Fig. 2. This set of structures, called as the MIAL structure below for convenience, was the one that has been confronted in great details with reactivity experiments. ${ }^{13,22}$

For the purpose of easy comparison, we also use this MIAL structure after the following brief analysis. ${ }^{23}$ A simple check is made by counting the change of the total nearestneighbor bonds with increasing cluster size. In general, the bond number should increase by at least 3 through adding one atom which is put simply on top of an atomic triangle without further relaxations to bring in even more bonds. This is true for most MIAL clusters, except $\mathrm{Ni}_{21}, \mathrm{Ni}_{29}, \mathrm{Ni}_{33}$, $\mathrm{Ni}_{37}, \mathrm{Ni}_{40}$, and $\mathrm{Ni}_{59}$ where the increase of bond number is equal to or less than 2 compared with $\mathrm{Ni}_{20}, \mathrm{Ni}_{28}, \mathrm{Ni}_{32}$, $\mathrm{Ni}_{36}, \mathrm{Ni}_{39}$, and $\mathrm{Ni}_{58}$, respectively. So, we suspect that these six MIAL structures are probably artifacts of the specific potential, or due to insufficient structural optimizations. We neglect these six members $\left(\mathrm{Ni}_{21}, \mathrm{Ni}_{29}, \mathrm{Ni}_{33}, \mathrm{Ni}_{37}, \mathrm{Ni}_{40}\right.$, and $\mathrm{Ni}_{59}$ ) from the MIAL list in our following discussions,

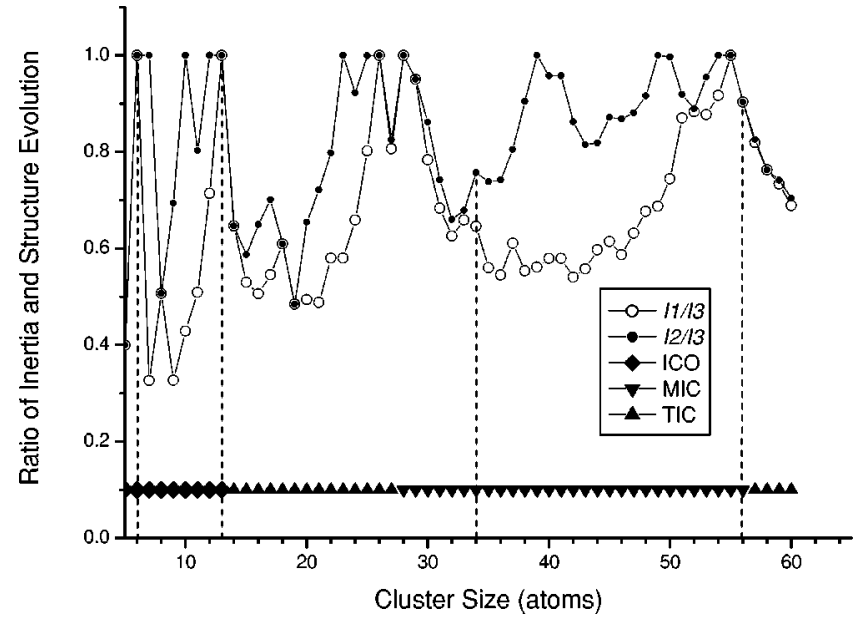

FIG. 2. Ratio of rotational inertia, $I_{1} / I_{3}$ and $I_{2} / I_{3}$, and the evolution of the MIAL structures. Four dashed lines show the experimental moment minima positions.

with the concern that possible structure uncertainty may mislead our understanding of nanocluster magnetism.

Symmetry, shape, and the size evolution are important for understanding the global behaviors of the delocalized electron states. For a simple and straight characterization of these properties, three principal axes and their corresponding rotational inertia (marked as $I_{1} \leqslant I_{2} \leqslant I_{3}$ ) are calculated for the MIAL clusters. Their ratios, $I_{1} / I_{3}$ and $I_{2} / I_{3}$, are plotted in Fig. 2. At $n=13,26,28$, and $55, I_{1} / I_{3}=I_{2} / I_{3}=1$, indicating that these structures are spherelike. In fact, $\mathrm{Ni}_{13}$ and $\mathrm{Ni}_{55}$ are completed icosahedral structures centering on one atom. On the other hand, the center of $\mathrm{Ni}_{28}$ is on an empty tetrahedral site. During the course of increasing size from one spherelike cluster to another one, i.e., from $n=13$ to 26 and 28 , or from $n=28$ to 55 , Ni clusters first become prolate ellipsoid (with $I_{3}>I_{2} \approx I_{1}$ ), and then change to oblate ellipsoid ( $I_{3}$ $\approx I_{2}>I_{1}$ ). This evolution of cluster shape leads to a drastic change of the degeneracy of the quantum confined delocalized $s$ states. As the results, the behaviors of filling these states deviate greatly from simple expectations based on a spherical jellium model (see discussions below in Sec. VI).

Two of the moment minima (i.e., $n=6$ and 13) on the size dependence of magnetic moment (vertical lines in Fig. 2) correspond exactly to the high-symmetry spherical shapes, while the other one at $n=56$ is pretty close to the highsymmetry structure at $n=55$. This hints to some relations between the moment minima and the cluster symmetries. However, the relation is not exact, and there is an exceptional minimum at $n=34$. Detailed theoretical calculations are necessary to clarify the true relation between the moment minima and the cluster symmetry

\section{MODEL HAMILTONIAN AND METHODOLOGY}

For transition metals, the TB Hamiltonian has been widely used because the interaction matrix elements can be easily parametrized to reproduce the $a b$ initio results in very good accuracy. Since the $s-p-d$ hybridization plays a major role in determining the magnetic properties of transition met- 
als, usually $3 d, 4 s$, and $4 p$ valence orbits are included, and the interaction matrix elements are parametrized by fitting the results to the equilibrium bulk bands. These parameters are then transferred to other nonperiodic systems, such as disordered solids, after proper scaling to account for the change of interatomic distance. However, for the cluster problems, one should be careful to include the surface effects, a crucial ingredient in the cluster systems, in the TB Hamiltonian.

Following Hamiltonian is used in present paper to describe the Ni clusters:

$$
\begin{aligned}
H= & {\left[\sum_{i L \sigma} \epsilon_{i l}^{0} c_{i L \sigma}^{\dagger} c_{i L \sigma}+\sum_{i j} \sum_{L L^{\prime} \sigma} t_{i j}^{L L^{\prime}} c_{i L \sigma^{\prime}}^{\dagger} c_{j L^{\prime} \sigma}\right]+H_{S O C}+H_{e e} } \\
& +\sum_{i^{\prime} \sigma}\left[\epsilon_{i^{\prime} s^{\prime}}^{0} c_{i^{\prime} s^{\prime} \sigma^{\prime}}^{\dagger} c_{i^{\prime} s^{\prime} \sigma^{\prime}}+t^{s s^{\prime}}\left(Z_{i^{\prime}}\right)\right. \\
& \left.\times\left(c_{i^{\prime} s^{\prime} \sigma^{\prime}}^{\dagger} c_{i^{\prime} s \sigma^{\prime}}+c_{i^{\prime} s \sigma^{\prime}}^{\dagger} c_{i^{\prime} s^{\prime} \sigma}\right)\right]+\sum_{i^{\prime} L \sigma} \Delta \epsilon_{i^{\prime}}\left(n_{s^{\prime}}^{i^{\prime}}\right) \\
& \times\left(c_{i^{\prime} L \sigma^{\prime}}^{\dagger} c_{i^{\prime} L \sigma}+c_{i^{\prime} s^{\prime} \sigma^{\prime}}^{\dagger} c_{i^{\prime} s^{\prime} \sigma}\right) .
\end{aligned}
$$

Here $\mathrm{c}_{i L \sigma}^{\dagger}\left(\mathrm{c}_{i L \sigma}\right)$ is the operator of the creation (annihilation) of an electron with spin $\sigma$ and orbital quantum number $L$ $=(l, m)$ at site $i$. Subscripts $i^{\prime}$ and $s^{\prime}$ represent, respectively, the surface atoms and an empty orbit attached to each surface atom, as explained below.

The first term in Eq. (1) represents the usual bulk bands through the bare orbital energies $\epsilon_{i l}^{0}$ and the interatomic hopping integrals $t_{i j}^{L L^{\prime}}$ between orbit $L$ at atom $i$ and orbit $L^{\prime}$ at its nearest-neighboring atom $j$. Values of these model parameters are taken from standard reference, ${ }^{24}$ which are obtained by fitting to LSDA bands of ferromagnetic fcc bulk Ni, in the Slater-Koster approximation, and taking only the two-center hopping integrals. Since the spin exchange will be considered in a separate interaction term $H_{e e}$, the parameters here (see Table II) are the average values for two spin channels listed in the reference book. ${ }^{24}$ As the interatomic distances in clusters are certainly not uniform and differ more or less from the bulk values, variation of the hopping integrals with the interatomic distance $r_{i j}$ is assumed to follow a power law $\left(r_{0} / r_{i j}\right)^{l+l^{\prime}+1}$, where $r_{0}$ is the bulk equilibrium distance and $l$ and $l^{\prime}$ are the orbital quantum numbers of the two orbits involved in the hopping. ${ }^{25}$

The second term $H_{S O C}$ is the spin-orbit coupling (SOC) given by

$$
H_{S O C}=\xi \sum_{i L \sigma, L^{\prime} \sigma^{\prime}}\left\langle L \sigma\left|\vec{S}_{i} \cdot \vec{L}_{i}\right| L^{\prime} \sigma^{\prime}\right\rangle c_{i L \sigma^{\prime}}^{\dagger} c_{i L^{\prime} \sigma^{\prime}},
$$

where the SOC strength is set as $\xi=0.073 \mathrm{eV}$ (Table II) for $d$ orbitals according to Ref. 26

The third term $H_{e e}$ is the intraatomic $d$-d electronelectron interaction, including both Coulomb and exchange interaction, and responsible for orbital and spin polarization. Since orbital polarization plays an important role in lowdimensional transition metal systems, following our previous work, ${ }^{27}$ we adopt the general concept of the LDA+U method $^{28}$ to account for it. In a generalized Hartree-Fock approximation including all possible pairings, the Hamiltonian reads

$$
H_{e e}=\sum_{i, L \sigma, L^{\prime} \sigma^{\prime}} V_{L \sigma, L^{\prime} \sigma^{\prime}}^{i} c_{i L \sigma^{\prime}}^{\dagger} c_{i L^{\prime} \sigma^{\prime}}
$$

where

$$
\begin{aligned}
V_{L \sigma, L^{\prime} \sigma^{\prime}}^{i}= & \sum_{L_{2} L_{3}}\left[\left[U_{L L_{2} L^{\prime} L_{3}} n_{L_{2} \bar{\sigma}, L_{3} \bar{\sigma}}^{i}\right.\right. \\
& \left.+\left(U_{L L_{2} L^{\prime} L_{3}}-U_{L L_{2} L_{3} L^{\prime}}\right) n_{L_{2} \sigma, L_{3} \sigma}^{i}\right] \delta_{\sigma \sigma^{\prime}} \\
& \left.-U_{L L_{2} L_{3} L^{\prime}} n_{L_{2}}^{i} \bar{\sigma}, L_{3} \sigma \delta_{\sigma \sigma^{\prime}}\right]-U\left(n^{i}-0.5\right) \delta_{L L^{\prime}} \delta_{\sigma \sigma^{\prime}} \\
& +J\left(n^{i \sigma}-0.5\right) \delta_{L L^{\prime}} \delta_{\sigma \sigma^{\prime}} .
\end{aligned}
$$

Here $n_{L \sigma, L^{\prime} \sigma^{\prime}}^{i}=\left\langle C_{i L \sigma}^{\dagger} C_{i L^{\prime} \sigma^{\prime}}\right\rangle$ is the single-site density matrix determined self-consistently, $n^{i \sigma}=\operatorname{Tr}\left[n_{L \sigma, L^{\prime} \sigma}^{i}\right]$ is the electron number of spin $\sigma, \bar{\sigma}$ means $-\sigma$, and $n^{i}=\Sigma_{\sigma} n^{i \sigma}$ is the total electron number on atom $i$. Being an extension of Eq. (5) of Ref. 28, present expression is rotationally invariant with respect to both space and spin.

Matrix elements $U_{L L_{2} L^{\prime} L_{3}}$ satisfy rotation summation relation as given by Eq. (6) of Ref. 28, and can all be determined by two parameters, namely, the average on-site Coulomb repulsion $U$ and the exchange $J$,

$$
\begin{gathered}
U=\frac{1}{(2 l+1)^{2}} \sum_{m m^{\prime}} U_{m m^{\prime} m m^{\prime}}, \\
U-J=\frac{1}{2 l(2 l+1)} \sum_{m m^{\prime}}\left(U_{m m^{\prime} m m^{\prime}}-U_{m m^{\prime} m^{\prime} m}\right) .
\end{gathered}
$$

In this formalism, the Stoner parameter, which determines the exchange splitting of the bulk bands, ${ }^{16}$ is $I=(2 l J$ $+U) /(2 l+1)$. To be compatible with the exchange splitting in the LSDA bulk bands, $I=1.12 \mathrm{eV}$ (Table II) is fixed in our calculations. However, the correlation parameter $U$ is often set adjustable, because its exact value may vary from system to system even for atoms of the same element, say, about 5 $\mathrm{eV}$ in oxides, but less than $3 \mathrm{eV}$ in metallic system. ${ }^{29} \mathrm{It}$ is also possible that, in the case of clusters, $U$ values could also be different for clusters with different sizes, or even be different for the center and boundary atoms in one single cluster. For simplicity, such variations are neglected in present study. We take $U=2.6 \mathrm{eV}$ in most calculations, and other values (1.8 and $3.2 \mathrm{eV}$, as in Table II) to check the influences of $U$ parameter variation.

Although terms 1-3 in Hamiltonian (1) mainly depict the physical behaviors exhibited in bulk systems, they could also describe, at least partly, the physical effects due to the coordination deficit of surface atoms. One example is the band narrowing, which transfers the $s$ electrons to more than half filled $d$ bands, leading to a surface moment reduction. This is well known from the earliest TB studies back to 1970s before the LSDA formalism. Later, however, LSDA revealed 
TABLE II. Parameters of Hamiltonian (1). Orbital energy, hopping integrals, SOC strength, and Stoner exchange are taken from standard references. Correlation parameter has been varied for comparison, and parameters for surface empty orbit and the surface valence shift have been chosen to give the most reasonable fit as stated in the text. All values are in units eV of except the dimensionless $Z_{\max }$.

\begin{tabular}{|c|c|c|c|c|c|c|}
\hline Orbital energy & $\begin{array}{c}\epsilon_{s}^{0} \\
15.50\end{array}$ & $\begin{array}{c}\epsilon_{p}^{0} \\
24.64\end{array}$ & $\begin{array}{c}\epsilon_{d}^{0} \\
9.74\end{array}$ & & & \\
\hline Hopping integral $\sigma$ & $\begin{array}{c}V_{s s \sigma} \\
-1.781\end{array}$ & $\begin{array}{l}V_{p p \sigma} \\
3.512\end{array}$ & $\begin{array}{c}V_{d d \sigma} \\
-0.667\end{array}$ & $\begin{array}{c}V_{s p \sigma} \\
2.256\end{array}$ & $\begin{array}{c}V_{s d \sigma} \\
-0.902\end{array}$ & $\begin{array}{c}V_{p d \sigma} \\
-1.237\end{array}$ \\
\hline Hopping integral $(\pi)$ & & $\begin{array}{l}V_{p p \pi} \\
0.345\end{array}$ & $\begin{array}{c}V_{d d \pi} \\
0.407\end{array}$ & & & $\begin{array}{c}V_{p d \pi} \\
0.266\end{array}$ \\
\hline Hopping integral $\delta$ & & & $\begin{array}{c}V_{d d \delta} \\
-0.037\end{array}$ & & & \\
\hline SOC strength $\xi$ & 0.073 & & & & & \\
\hline Stoner exchange $I$ & 1.12 & & & & & \\
\hline Correlation $U$ & \multicolumn{3}{|c|}{ 2.6, and with options 1.8, and 3.2} & & & \\
\hline Surface empty orbit & $\begin{array}{c}\epsilon_{s^{\prime}}^{0} \\
15.50\end{array}$ & $\begin{array}{c}V_{s s^{\prime} \sigma} \\
-2.460\end{array}$ & $\begin{array}{c}Z_{\max } \\
15.8\end{array}$ & & & \\
\hline Surface valence shift $\chi$ & 2.3 & & & & & \\
\hline
\end{tabular}

that giant moments exist on $3 d$ transition metal surfaces, which was subsequently confirmed by experiments. 5 The physical origin for this giant moment on surface is the energy shift of the electronic orbits of surface atom, which lowers the electron occupancy and increase the number of $d$ holes on the surface atoms. This shift is due to the spillover of surface electrons and the dipole layer thus formed on the surfaces, which has been treated properly by standard all electron LSDA calculations for Ni systems in the film geometry (see Table I for the core-level shift). However, to include properly this energy shift in a TB study of the nanocluster magnetism, where the surface is much more complex than in the film geometry, is obviously a key problem, but a toughest challenge.

To account for the electron spillover existing on cluster surfaces, following Weissmann's group ${ }^{12}$, we introduce an extra orbit $s^{\prime}$ to each surface atom $i^{\prime}$ (atom with coordination number $Z<12$ for Ni clusters), and add the fourth term to our Hamiltonian (1). We assume this orbit $s^{\prime}$ to locate in the vacuum outside the surface, has an $s$ symmetry, an energy $\epsilon_{s^{\prime}}^{0}$, and interact with $s$ orbit of the same surface atom by hopping integral $t^{s s^{\prime}}$. Electron occupation of this $s^{\prime}$ orbit represents the spillover of electrons from surface atoms to the vacuum. In the ideal film geometry as treated by Weissmann's group, all surface atoms are identical, and the orbital energy and hopping integrals of $s^{\prime}$ orbit are constants. To account for the differences of environments encountered by atoms on cluster surfaces, variations should be introduced.

The smaller the local coordination, the larger the open space of this surface atom. This change of open space volume could be accounted for approximately by putting the hopping integral proportional to the square root of the number of coordinate deficit,

$$
t^{s s^{\prime}}(Z)=V_{s s^{\prime} \sigma} \sqrt{Z_{\max }-Z} .
$$

Here $V_{s s^{\prime} \sigma}$ is the hopping strength between $s$ and $s^{\prime}$ orbits, $Z_{\max }$ is approximately the maximum allowable number of nearest-neighbor spheres, and $Z_{\max }-Z$ is thus the number of empty volume around an atom with coordination number $Z$. Though $Z_{\max }=12$ seems to be a natural choice for Ni which exhibits equilibrium fcc bulk structure, we prefer to take both $V_{s s^{\prime} \sigma}$ and $Z_{\text {max }}$ as adjustable parameters, while keeping $\epsilon_{s^{\prime}}^{0}$ constant and $\epsilon_{s^{\prime}}^{0}=\epsilon_{s}^{0}$ for simplicity. The parameter values, which give the most reasonable moment variation with respect to the coordination number of atoms, are listed also in Table II.

After considering the spillover of electrons, Weissmann and co-workers, ${ }^{12}$ introduced an intersite Coulomb term to describe the valence level shift,

$$
\Delta \epsilon_{i}=\sum_{j} \frac{U}{1+U\left|R_{i}-R_{j}\right|} \Delta n_{j} .
$$

Equation (8) returns to the intra-atomic Coulomb repulsion as $R_{i}=R_{j}$, and represents a bare Coulomb interaction without any screening when atom $j$ is far from atom $i$. However, considering that the spillover of electrons on the surface may happen in a length scale longer than an atomic radius, we think the interaction of the spilled electron might be different from the usual intraatomic electron-electron interaction, and the screening could be very strong for metallic materials. As shown in the film calculations, both the charge and potential variations are localized on the very top layer. We thus assume, in another extreme of strong screening, that the orbital shifts are localized only on the surface atoms of a cluster. We adopt a term

$$
\Delta \epsilon_{i^{\prime}}\left(n_{s^{\prime}}^{i^{\prime}}\right)=\chi n_{s^{\prime}}^{i^{\prime}}, \quad i^{\prime} \in \text { surface, }
$$

for this surface valence orbital shift, i.e., electron spillover only changes the potential right on the surface atom. This shift of orbital energy affects all valence $(3 d, 4 s$, and $4 p)$ orbits and the empty orbits $s^{\prime}$ itself [see Hamiltonian (1)]. Proportional parameter $\chi=2.3 \mathrm{eV}$ (Table II) is found to give the best fit of the surface valence orbital shift, calculated in 
present paper for clusters, to the core level shift obtained in the film geometry by LSDA calculations (see Sec. V A below).

Structures of very small clusters $(n=2-8)$ are very open, with average coordination number less than 5. Parametrization used above for the description of surface effects may lead to larger deviations. Results presented below are only for clusters with $n \geqslant 9$.

Let us now describe the process of going to full selfconsistency in present calculations. First, by switching off the exchange, correlation and the spin-orbit coupling, i.e., setting $I=U=\xi=0$, we get a density matrix containing the effect of electron transfer between atoms but without spin and orbital polarization. Then $I, U$, and $\xi$ are turned on, and iterations continue by adding a finite uniform diagonal spin polarization, i.e., $\Delta_{\sigma}$ for spin $\sigma$ up, $-\Delta_{\sigma}$ for spin $\sigma$ down, to the nonpolarized density-matrix elements of all $d$ orbits. Self-consistency is achieved by solving Hamiltonian equation (1) iteratively. From the self-consistent density matrix, spin magnetic moment $\mu_{\text {spin }}$ and orbital magnetic moment $\mu_{\text {orb }}$ are obtained from a vector average over the atomic moments:

$$
\begin{aligned}
& \vec{\mu}_{\text {orb }}=\frac{1}{n} \sum_{i} \vec{\mu}_{\text {orb }}^{i}=\frac{1}{n} \sum_{i} \sum_{L \sigma, L^{\prime} \sigma^{\prime}} n_{L \sigma, L^{\prime} \sigma^{\prime}}^{i}(\vec{L})_{L L^{\prime}} \delta_{\sigma \sigma^{\prime}}, \\
& \vec{\mu}_{\text {spin }}=\frac{1}{n} \sum_{i} \vec{\mu}_{\text {spin }}^{i}=\frac{1}{n} \sum_{i} \sum_{L \sigma, L^{\prime} \sigma^{\prime}} n_{L \sigma, L^{\prime} \sigma^{\prime}}^{i}(2 \vec{S})_{\sigma \sigma^{\prime}} \delta_{L L^{\prime}}
\end{aligned}
$$

The total moment $\vec{\mu}$ is then calculated as their vector sum.

Although our iterations start from a uniform distribution of small spin polarization and vanishing orbital polarization, the physical spin and orbital moments obtained by selfconsistently solving Hamiltonian (1) could be nonuniform and noncollinear. However, it is found that the noncollinearity is very weak.

Although SOC could generate anisotropy, we did not search for the energy minimum over all possible directions, but only compared calculations with spin along three principal axes of the clusters. We found that the energy differences are typically less than $0.005 \mathrm{eV} /$ atom. The effect of SOC on moment values is almost negligible, and the moment differences for three directions are less than $0.02 \mu_{B}$ /atom. Results reported below are calculated by setting the spin along the largest inertia axis.

\section{IMPORTANCE OF ORBITAL POLARIZATION}

In this section, we first separately discuss the spin and orbital contributions to the total moments in connection with previous theoretical treatments, and then compare our results with experimental data. We show that orbital moments are enhanced by over an order of magnitude for surface atoms in nanoclusters, and dominate the oscillations of measured magnetic moment versus cluster size. In contrast to bulk crystals where theoretically calculated spin moments agree well with the measured total moments, orbital polarization is

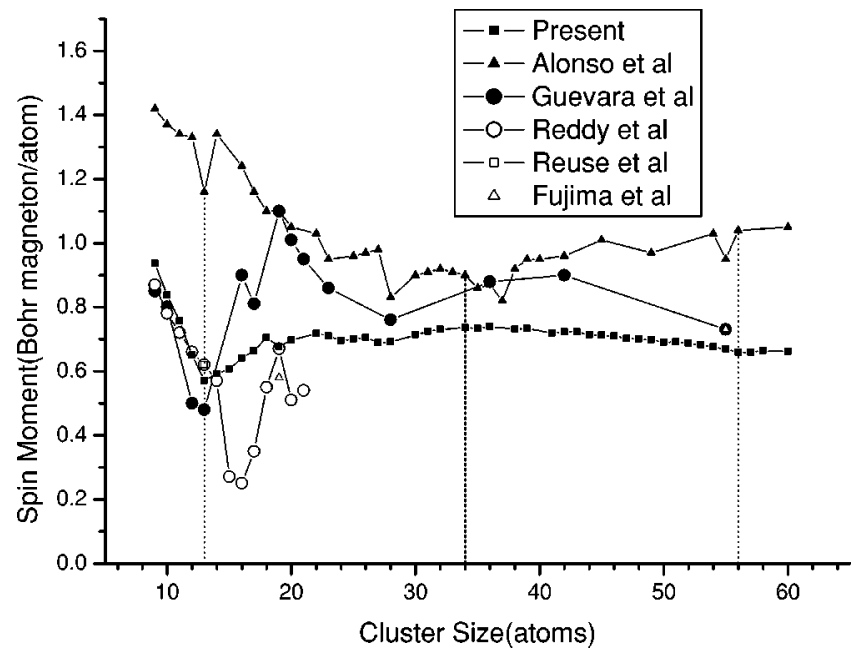

FIG. 3. Spin moments of clusters with different sizes in present calculations (square), showing good agreements with three firstprinciples calculations (open symbols) for most clusters, except for $n=14,15,16,17,18$, and 20, when different structures are used as explained in the text. Two other TB calculation results (solid symbols) are also plotted for comparison. Three vertical dashed lines show the experimental moment minima positions.

crucial to reach agreements with experiments in nanoclusters.

\section{A. Size dependence of spin moment}

Spin moments calculated by Hamiltonian (1) are found almost the same as those obtained by switching off orbital correlation and SOC, i.e., setting $U=0$, and $\xi=0$, but keeping Stoner parameter $I=1.12 \mathrm{eV}$ unchanged. One can compare our spin moments with previous theoretical results of both LSDA and TB calculations, all of which did not consider orbital correlation and SOC and give only spin moment. Comparison with first-principles results serves as a critical justification of our TB parametrization scheme.

Figure 3 compares our results with those given by Reddy et al., ${ }^{10}$ who, using first-principles molecular-orbital theory, studied the magnetic properties of Ni clusters in most detail. For $\mathrm{Ni}_{n}$ with $n=9-14$, and $n=19$, our $\mu_{\text {spin }}$ 's are in perfect agreement with their results. Either Reddy et al.'s or present spin moments drop rapidly for small clusters from $n=9$ to 13, in accordance with experiments (Fig. 1), but both calculated values are obviously lower than experimental results. However, disagreements exist for $n=15-18$, and $n=20$, where our $\mu_{\text {spin }}$ 's are larger than Reddy et al. by about $0.2-0.4 \mu_{B} /$ atom. In order to understand the origin of this discrepancy, we checked the structures used in both calculations, and show the coordination in Table III. It is found that for $n=9$ through 14, and $n=19$, the MIAL structure used in present calculation is exactly the same as that used by Reddy et al., but for $n=15,16,17,18$, and 20, there is a big difference. We conclude that all differences between present results and Reddy et al. are not from the theoretical methods, though one is a LSDA and the other is a TB calculation, but from the structures used in two calculations. 
TABLE III. Comparison between the structures used by Reddy et al. and the MIAL structures for clusters $\mathrm{Ni}_{n}$ with $n=9-20$. Listed are the number of atoms with coordination number $Z$ in parentheses, and the number of total bonds.

\begin{tabular}{lcccc}
\hline \hline$n$ & $\begin{array}{c}\text { Reddy et al. } \\
\text { Atoms }(Z)\end{array}$ & Bonds & MIAL & Btoms \\
\hline 9 & $4(4), 2(5), 2(6), 1(8)$ & 23 & $4(4), 2(5), 2(6), 1(8)$ & 23 \\
10 & $3(4), 3(5), 3(6), 1(9)$ & 27 & $3(4), 3(5), 3(6), 1(9)$ & 27 \\
11 & $2(4), 4(5), 4(6), 1(10)$ & 31 & $2(4), 4(5), 4(6), 1(10)$ & 31 \\
12 & $5(5), 6(6), 1(11)$ & 36 & $5(5), 6(6), 1(11)$ & 36 \\
13 & $12(6), 1(12)$ & 42 & $12(6), 1(12)$ & 42 \\
14 & $1(3), 9(6), 3(7), 1(12)$ & 45 & $1(3), 9(6), 3(7), 1(12)$ & 45 \\
15 & $12(6), 2(7), 1(14)$ & 50 & $2(4), 8(6), 2(7), 2(8), 1(12)$ & 49 \\
16 & $1(4), 7(5), 7(6), 1(9)$ & 45 & $2(4), 1(5), 7(6), 2(7), 2(8), 1(9), 1(12)$ & 53 \\
17 & $2(4), 3(5), 11(6), 1(11)$ & 50 & $2(4), 2(5), 6(6), 2(7), 3(8), 1(10), 1(12)$ & 57 \\
18 & $2(4), 8(5), 8(7)$ & 52 & $5(5), 6(6), 5(8), 1(11), 1(12)$ & 62 \\
19 & $12(6), 5(8), 2(12)$ & 68 & $12(6), 5(8), 2(12)$ & 68 \\
20 & $2(5), 16(6), 2(11)$ & 64 & $1(4), 10(6), 2(7), 3(8), 2(9), 2(12)$ & 72 \\
\hline \hline
\end{tabular}

First-principles calculations have been made also by other authors on a few high-symmetry clusters (Fig. 3). Using linear combination of atomic molecular-orbital approach within the density-functional formalism, Reuse et al. ${ }^{9}$ get a magnetic moment $0.62 \mu_{B}$ /atom for $\mathrm{Ni}_{13}$. Also, using the spinpolarized discrete variational $X \alpha$ method, Fujima and Yamaguchi obtained the magnetic moments 0.58 and 0.73 $\mu_{B}$ /atom for $\mathrm{Ni}_{19}$ and $\mathrm{Ni}_{55}$, respectively. ${ }^{11}$ All these results are in good agreement with present ones, $\mu_{\text {spin }}=0.57,0.68$, and $0.66 \mu_{B}$ /atom for $n=13,19$, and 55 .

We conclude at this point that with present TB formalism and parametrization scheme, the calculated spin moments are in very good agreement with $a b$ initio results within an error bar about $0.1 \mu_{B}$ /atom, as long as the calculations are made for the same geometrical structures. This gives us confidences to extend our TB studies towards large clusters which are hard for direct first-principles calculations. It also shows that a good geometrical optimization is crucial when a detailed comparison is to be made with experiments, irrespective of theoretical approaches used in the studies of cluster magnetism.

We also note that all first-principles as well as our spin moments are significantly lower than experimentally measured values in a rather wide size range. Our spin moments do show a minimum right at $n=13$, but it is not as sharp as the experimental one. Even more unexpected is that, contrary to experimental finding, our spin moment shows very smooth size variations without oscillations when $n \geqslant 22$, in obvious disagreements with experiments where two minima exist at $n=34$ and 56 (vertical line in Fig. 3). We demonstrate below that these discrepancies can be resolved when the total moments, instead of the spin moments only, are compared with the experimental moment values.

Before going to a discussion about the orbital contribution given in present formalism, it is worth to compare also our results of spin moment with two other important TB calculations. Since cluster magnetism has been studied by Alonso and co-workers ${ }^{13-15}$ using exactly the same MIAL geometry, it is thus of special importance for us to compare with their results, in order to evaluate the influences of different approximations in the adopted Hamiltonian. Alonso co-workers have been able to make their spin magnetic moments significantly larger than ours and other LSDA results (Fig. 3). In their calculations, an orbital energy shift $\Omega_{i l}$ was introduced, which depends not only on atom (subscript $i$ ) but also on orbit (subscript $l$ ). These parameters were adjusted to make the number of $s, p$, and $d$ electrons on each atom equal to values preassigned according to its coordination number through a linear interpolation between the isolated atoms and atoms in bulk crystals. While this procedure approximately accounted for the local surface effect, it completely neglected the redistribution of electrons between $s$ and $d$ orbits which may happen due to the global volume confinement. Besides, the valence energies of different $l$ 's were shifted so differently (even with different signs), which is hard to understand on a Coulomb mechanism. Thus Alonso and coworkers' result of larger $\mu_{\text {spin }}$, though closer to the measured total moment, was more or less an artifact, depending on the preassignment of the number of electrons. In addition, compared with experiments, their results did not present the right positions (vertical lines in Fig. 3) of moment minima too.

In another TB calculation, Guevera et al. ${ }^{12}$ studied the ideally cut fcc Ni clusters. They used a Hamiltonian similar to ours, i.e., adding $s^{\prime}$ orbits to surface atoms to consider the electron spillover. In most cases, our results agree well with those of Guevera et al. (Fig. 3). Again, the spin moments by Guevara et al. were obviously lower than experimental ones, similar to present results and other LSDA calculations. Since Guevera et al. did not consider a complete series of cluster size, it is not possible to check the moment minima positions to compare with experiments.

At this point, we come to a conclusion that the measured magnetic moments cannot be considered as only the spin moments, even though the calculated spin moments do show 


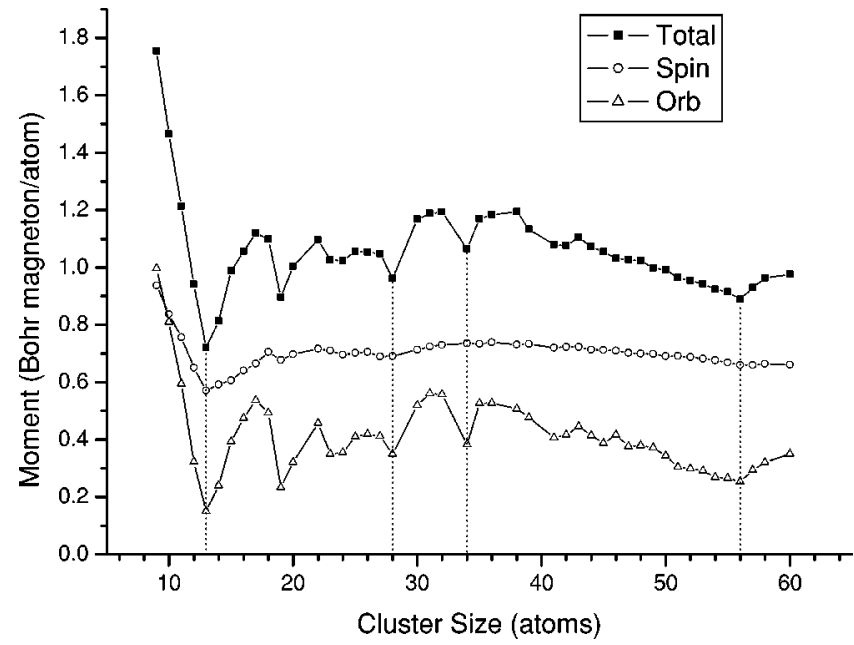

FIG. 4. Calculated spin, orbital, and total magnetic moments of Ni clusters. Four vertical dashed lines show present theoretical moment minima positions.

enhancements in small clusters. The prominent differences between measured and calculated moments have to find other explanations.

\section{B. Orbital moment and its size dependence}

In principle, both spin and orbital magnetic moment have contribution to the total magnetic moment in any system, though the orbital one has been mostly quenched in bulk crystals. However, it is shown that at the low dimension, ${ }^{16-19}$ the orbital quenching could be released, and orbital polarization appears prominently. In order to compare spin and orbital contributions, we plot spin, orbital, and total moments together in Fig. 4, as the functions of cluster size. For $\mathrm{Ni}_{n}$ with $22 \leqslant n \leqslant 60, \mu_{o r b}$ varies between 0.3 and $0.6 \mu_{B}$ /atom. Compared with the bulk value, $\mu_{\text {orb }}$ has been enhanced over an order of magnitude. Decreasing further the cluster size, $\mu_{\text {orb }}$ increases even more. For example, $\mu_{\text {orb }}$ of $\mathrm{Ni}_{9}$ reaches about $1.0 \mu_{B}$ /atom. We note that variations in orbital moments versues cluster size are much larger than in spin moments. As seen from Fig. 4, the oscillations of total magnetic moment mainly come from the orbital contribution, which generates several minima in this range of cluster size. Even at $\mathrm{Ni}_{13}$, where both spin and orbital magnetic moments contribute to the sharp minimum, the orbital contribution is still the dominant one.

Of course, orbital moments calculated above depend on the choice of the correlation parameter $U$. Unfortunately, there is no standard value for $U$, and it is also uncertain how much it depends on the cluster size, and how it varies from one atom to another even in one single cluster. To ensure that our physical conclusions drawn below are not limited to this particular choice of parameter, i.e., $U=2.6 \mathrm{eV}$, calculations are made also with $U=1.8$ and $3.2 \mathrm{eV}$, in order to cover a wider range of generally accepted values of correlation. Since the Stoner exchange is kept as a constant, calculated spin moments change only slightly (less than $0.1 \mu_{B}$ /atom) when $U$ changes from 1.8 to $3.2 \mathrm{eV}$. Results of orbital moments are shown in Fig. 5 for comparison. As expected,

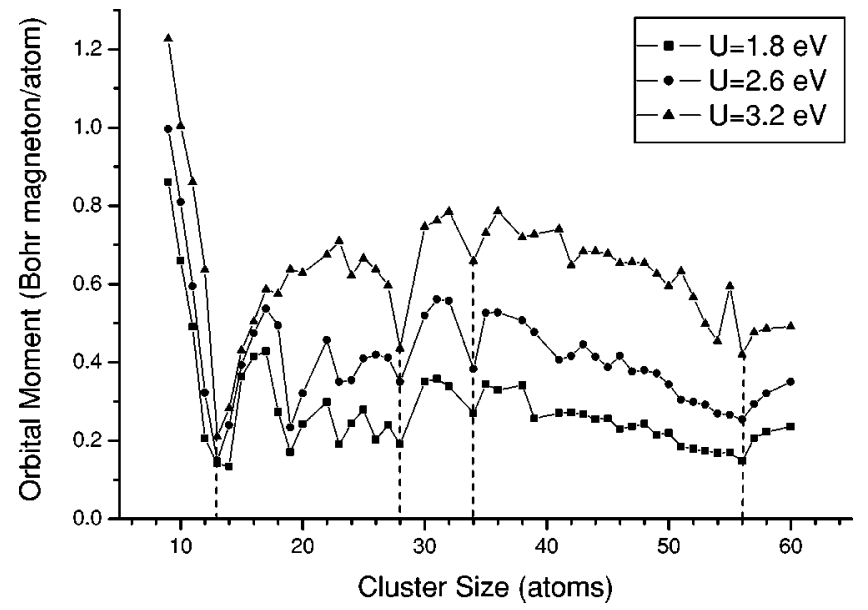

FIG. 5. Orbital moments calculated for correlation parameters $U=1.80,2.60$, and $3.20 \mathrm{eV}$. Four vertical dashed lines show present theoretical moment minima positions, which exist independent of the value of $U$ parameter in this range.

larger $U$ gives stronger orbital polarization. Approximately, for $n=22-60$, orbital moments are $0.2-0.3,0.3-0.6$, and 0.4-0.8 $\mu_{B}$ /atom for $U=1.8,2.6$, and $3.2 \mathrm{eV}$, respectively. Among these three parameter values, $U=2.6 \mathrm{eV}$ gives the best fit to experimental results over the entire range of cluster size (see the following section).

However, not all featured minima positions coincide each other for all three parameter values. Only four exhibit independence on the choice of $U$ parameter within the range from 1.8 to $3.2 \mathrm{eV}$, namely, the moment minima at or near $n$ $=13,28,34$, and 56 (vertical dashed lines in Fig. 5). We will discuss the physical origin in following sections. The remaining three minima at $n=19,23$, and 40 exist only for $U=2.6 \mathrm{eV}$, but may disappear upon the change of $U$ value. We have no reason to expect them appearing definitely in experiments, and do not consider them in our further discussions.

\section{General comparison with experiments}

The total magnetic moments are plotted in Fig. 6 for $\mathrm{Ni}$ clusters with $n=9-60$, compared with the experimental results of Apsel et al. ${ }^{3}$ and Knickelbein. ${ }^{4}$ Our calculations reproduce many features given by the two experiments, and the magnetic moment enhancement over the bulk values $\left(0.61 \mu_{B}\right)$.

In the region between $n=9$ and 28, our results are in good agreements with Knickelbein's experimental results, with differences typically less than $0.1 \mu_{B}$ /atom. Our results show the same trend of size dependence as Apsel et al. from $n=12$ to 20 , though our total moments are smaller by about 0.2-0.4 $\mu_{B}$ /atom. In particular, both calculated and the two experimental results show the pronounced sharp minimum at $\mathrm{Ni}_{13}$, and the significant moment increases as the cluster size decreases to below $n=13$.

For $\mathrm{Ni}_{n}$ with $30 \leqslant n \leqslant 38$, our results are larger (about $0.1 \sim 0.2 \mu_{B}$ /atom) than those of Apsel et al., but show correctly the minimum at $\mathrm{Ni}_{34}$. For $\mathrm{Ni}_{n}$ with $40 \leqslant n \leqslant 60$, our 


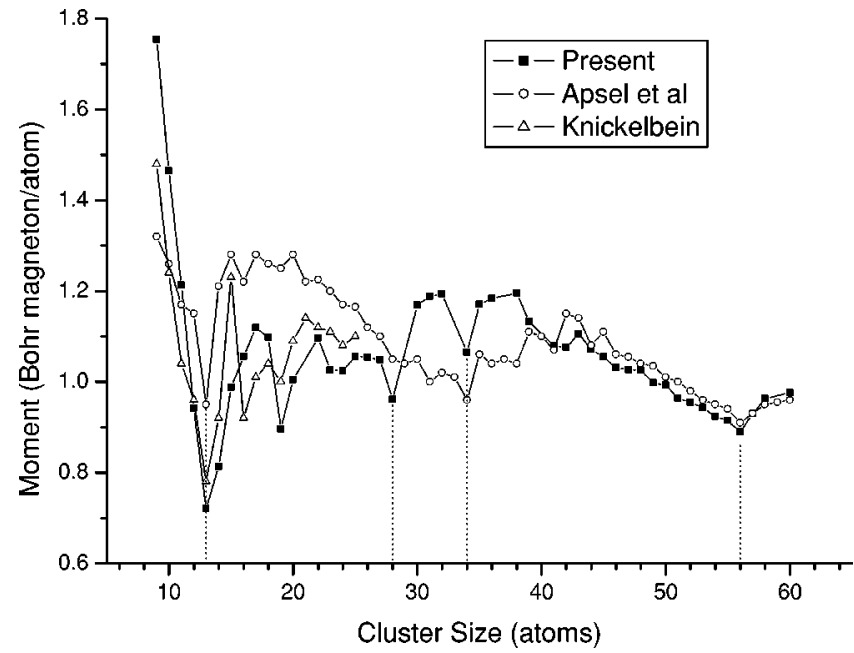

FIG. 6. Comparison of calculated total moment of Ni clusters with the experimental values by Apsel et al. and Knickelbein. Vertical dashed lines show the theoretical moment minima positions.

results are in excellent quantitative agreement with Apsel et al. within $\pm 0.05 \mu_{B}$ /atom. The moment variation in this region is very smooth, with only one minimum at $n=56$, also in agreement with Apsel et al. A minimum at $n=28$ exists in our calculations, but is not observed experimentally. Because there is a structure transformation from MIC to TIC right at $n=27-28$ (Fig. 2), the MIC structure used in the present calculation is obviously not far from other metastable TIC structures. A further check of the stability of the MIC structure of $\mathrm{Ni}_{28}$ has to be made to clarify this discrepancy.

As shown in previous sections, the spin moments given by either $a b$ initio or TB calculations are much smaller than the experimentally measured total moment, and do not exhibit the correct oscillatory size dependence. After considering orbital correlation and SOC, our calculations have reproduced not only the general trend of moment's size dependence including all minima located at $\mathrm{Ni}_{13}, \mathrm{Ni}_{34}$, and $\mathrm{Ni}_{56}$, but also the absolute moment values measured in experiments. This agreement shows clearly that orbital contribution is an indispensable part in nanocluster magnetism, because not only $\mu_{\text {orb }}$ has been greatly enhanced and compares fairly with its spin counterpart, but also the moment oscillations are mainly due to the orbital contribution.

\section{SURFACE ENHANCEMENT}

In this section, we show that not only the spin moment, but also orbital moment are enhanced for the surface atoms of the clusters due to the change of their local environment. While the spin enhancement is, as well known from previous studies on the magnetism of ultrathin films, due to surface valence orbital shift and the increase of $d$ band holes induced by this shift, an enhancement for the orbital contribution has also been induced by this increase of $d$ holes, and is found even stronger. This plays an important role in determining the size dependence of the measured total moment, especially in the range of cluster size larger than 20 atoms.
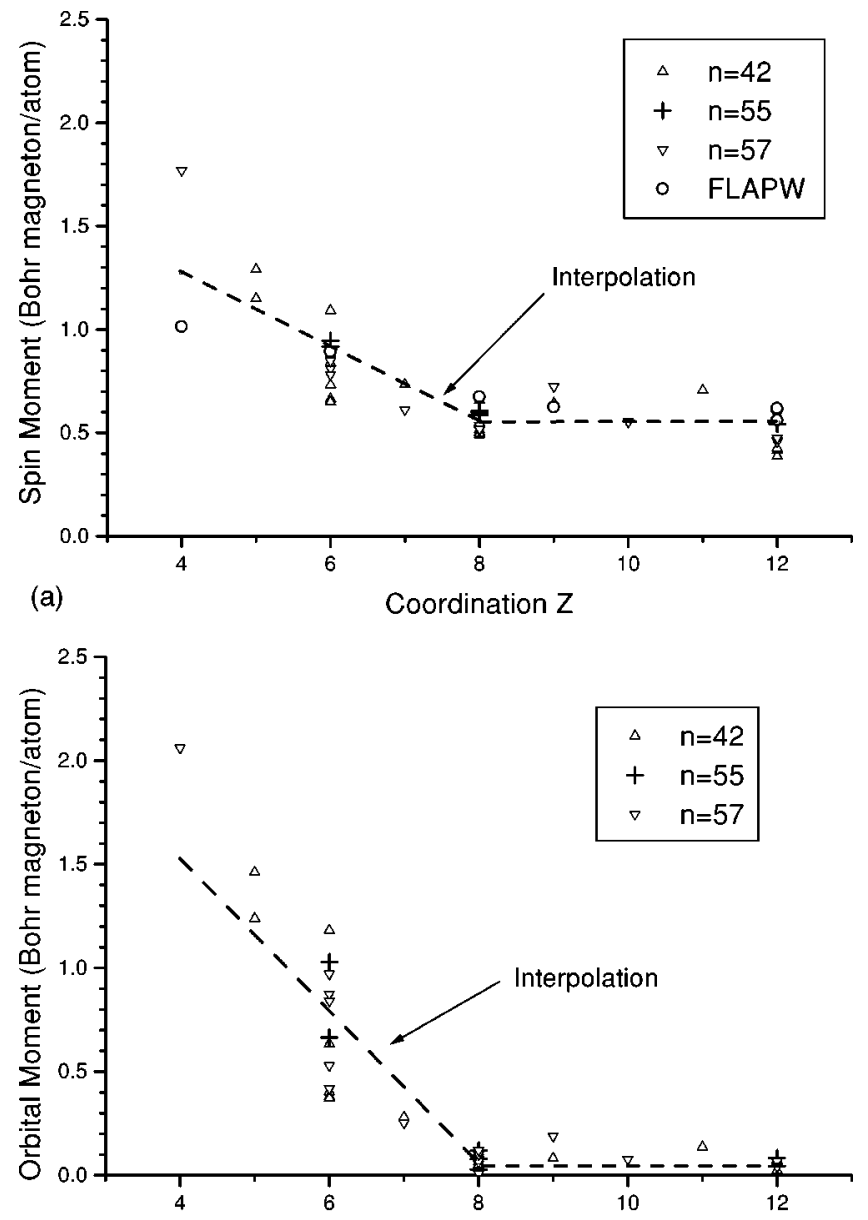

(b)

Coordination Z

FIG. 7. Dependence of local spin (a), and orbital (b) moment on the coordination number. Dashed lines represent an interpolation between an isolated atom and that in a bulk crystal as given by Eq. (11) in the text. Open circles denote the results of Ni films calculated by the standard FLAPW method as given in Table I.

\section{A. Dependence on coordination}

Although there are no experimental techniques to probe the local magnetic moment distribution in a cluster, it is still very interesting to analyze the contributions from different sites inside a cluster. This will help us to understand the size dependences of spin and orbital magnetic moments, and provide further insights on the enhancements of $\mu_{\text {spin }}$ and $\mu_{\text {orb }}$ in nanoclusters. To check the suggestion of Jensen and Bennemann, we plot in Figs. 7(a) and 7(b), respectively the spin and orbital magnetic moments of all atoms inside three clusters- $\mathrm{Ni}_{42}, \mathrm{Ni}_{55}$, and $\mathrm{Ni}_{57}$, as the functions of their coordination numbers $Z$. We chose these three clusters because our calculations yield the best agreements with experiments in that size region (Fig. 6). In addition, they cover a wide range of sampling by containing atoms with $Z=4-12$, and exhibiting oblate, spherical, as well as prolate ellipsoids (Fig. 2).

Consider first the atoms with $Z=12$. The symmetry of $\mathrm{Ni}_{55}$ is very high, possessing 13 atoms with $Z=12$, split into three nonequivalent types after including SOC. For these atoms, we find $\mu_{\text {spin }} \approx 0.54 \mu_{B} /$ atom, and $\mu_{\text {orb }}$ 
$\approx 0.06 \mu_{B}$ /atom [Figs. $7(\mathrm{a})$ and $7(\mathrm{~b})$ ] showing orbital quenching similar to that in the bulk fec Ni crystal. Although the symmetries of $\mathrm{Ni}_{42}$ and $\mathrm{Ni}_{57}$ are different from that of $\mathrm{Ni}_{55}$, atoms with $Z=12$ show similar moment values since the $d$ orbits of $\mathrm{Ni}$ atoms are very local. The agreement with the corresponding bulk values $\mu_{\text {spin }} \approx 0.55 \mu_{B}$ and $\mu_{\text {orb }}$ $\approx 0.05 \mu_{B},{ }^{30}$ is another evidence to show that our Hamiltonian parameters, namely, the $U, I$, and $\xi$ are chosen reasonably.

We next consider $Z=8$ atoms. There are 30 such atoms in $\mathrm{Ni}_{55}$, again split into three nonequivalent types after including SOC. As shown in Figs. 7(a) and 7(b), their spin and orbital moment differ not too much from those of $Z=12$ atoms. It is also true for the $Z=8$ atoms in $\mathrm{Ni}_{42}$ and $\mathrm{Ni}_{57}$.

Finally, there are 12 atoms with $Z=6$ in $\mathrm{Ni}_{55}$, which are sorted into two nonequivalent types after including SOC. These atoms have $\mu_{\text {spin }} \approx 0.93 \mu_{B} /$ atom and $\mu_{\text {orb }}$ $\approx 0.7-1.0 \mu_{B}$ /atom, both obviously enhanced from the bulk values [Figs. 7(a) and 7(b)].

Lower-symmetry clusters $\mathrm{Ni}_{42}$ and $\mathrm{Ni}_{57}$ contain more types of atoms. Atoms with even the same coordination could be different by relaxation of bond length or bond angle, etc. Due to this change of environments, sites with the same coordination number may have different $\mu_{\text {spin }}$ or $\mu_{\text {orb }}$, and this difference could be quite large in low symmetry clusters [Figs. 7(a) and 7(b)]. Nevertheless, as shown obviously in the figures, lower coordination number always results in larger local spin and orbital magnetic moments.

For the sites with $Z \geqslant 8$ (i.e. bulk and strongly bonding surface atoms), both $\mu_{\text {spin }}$ and $\mu_{\text {orb }}$ are not enhanced appreciably. On the contrary, for the sites with $Z \leqslant 6$ (i.e., weakly bonding surface atoms), both $\mu_{\text {spin }}$ and $\mu_{\text {orb }}$ are greatly enhanced. This sharp distinction could be understood from the general argument of Ref. 16, that for a given ratio between the orbital correlation and atomic bonding strength, the ground state changes from an orbital polarized one to an orbital quenched one at a certain dimensionality or coordination number.

Although both local spin and orbital magnetic moments increase with decreasing coordination, the enhancement of spin moment is smaller than in orbital one. For example, while spin moment increases from $0.55 \mu_{B}$ /atom for $Z$ $=12$ to $0.7-1.2 \mu_{B}$ /atom for $Z=6$, orbital moment increases from $0.05 \mu_{B}$ /atom to $0.4-1.2 \mu_{B} /$ atom, over an order of magnitude. We note that the magnitude of orbital moment change is $0.35-1.15 \mu_{B}$ /atom, approximately twice as large as that for spin, $0.15-0.65 \mu_{B}$ /atom. This partly explains the stronger oscillations in $\mu_{\text {orb }}$.

As a numerical recipe, we found that the local spin and orbital magnetic moments versus coordination can be approximated by

$\mu_{\text {spin }(\text { orb })}(Z)= \begin{cases}\mu_{\text {spin }(\text { orb })}^{\text {bulk }}, & Z \geqslant 8 \\ \frac{1}{8}\left[(8-Z) \mu_{\text {spin }(o r b)}^{\text {atom }}+Z \mu_{\text {spin }(o r b)}^{\text {bulk }}\right], & Z<8\end{cases}$

where $\mu_{\text {spin }}^{\text {atom }}=2.00 \mu_{B}$ and $\mu_{\text {orb }}^{\text {atom }}=3.00 \mu_{B}$ are, taken from the Hund's rule values for $\mathrm{Ni}$ atoms, and $\mu_{\text {spin }}^{\text {bulk }}=0.55 \mu_{B}$ and

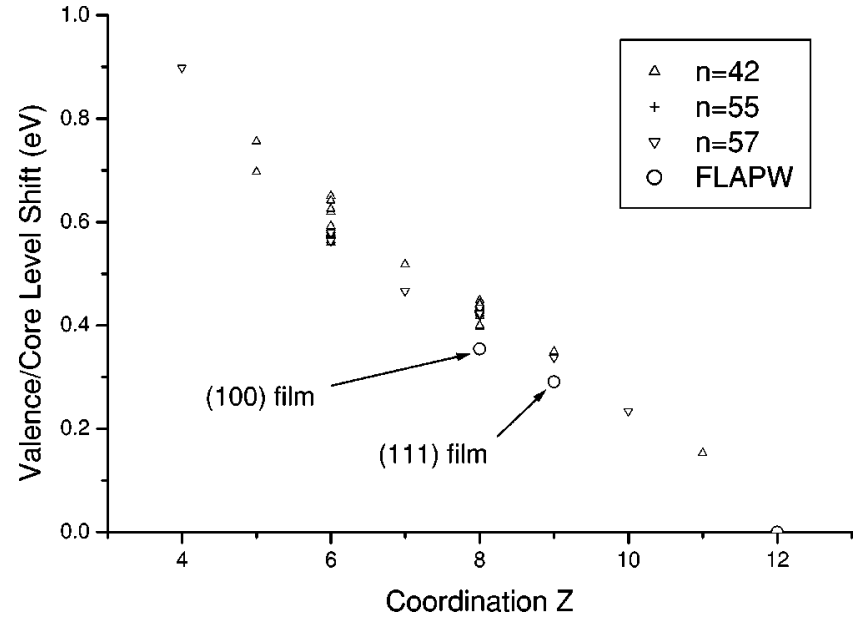

FIG. 8. Valence orbital shift as a function of the coordination number, compared with the core level shift (circles) of surface atoms from standard FLAPW film calculations as given in Table I.

$\mu_{\text {orb }}^{\text {bulk }}=0.05 \mu_{B}$ are taken from the experimental values of bulk Ni crystals. The interpolation results calculated by Eq. (11) are shown in Figs. 7(a) and 7(b) by dashed lines. It is worth mentioning that $Z$-interpolation equation (11) holds approximately for all $\mathrm{Ni}_{n}$ clusters with $n$ larger than 22, although it is derived from $\mathrm{Ni}_{42}, \mathrm{Ni}_{55}$, and $\mathrm{Ni}_{57}$ only.

Coordination deficit of surface atoms is known to have two opposite effects to the spin magnetism, i.e., the band narrowing which leads to a spin moment decrease for over half filled $3 d$ metals, and the upward shift of surface valence orbits due to the dipoles formed by the surface electronic spillover. It is the latter effect which surpasses the former one, leading to an overall giant surface moment, first revealed in early 1980s by LSDA calculation. ${ }^{5}$ Figure 8 depicts the calculated surface valence orbital shifts for $\mathrm{Ni}_{42}, \mathrm{Ni}_{55}$, and $\mathrm{Ni}_{57}$ as the functions of the corresponding coordination numbers. It is shown that the valence orbital shift is not sensitive to the cluster size, but depends mainly on the coordination number. Because a site with lower $Z$ has more $s^{\prime}$ occupation, the shift increases almost linearly with the local coordination deficit.

This valence orbital shift obtained in clusters by our TB calculations could be compared with the core level shifts obtained in films by standard LSDA calculations, because both of them are due to the electron spillover and have the same physical origin. For atoms on $\mathrm{Ni}$ (100) and (111) surfaces, their local coordination numbers are 8 and 9, respectively. According to standard FLAPW calculations made for five-layer slabs, ${ }^{6}$ the corresponding $3 p_{3 / 2}$ core-level shifts are 0.35 and $0.29 \mathrm{eV}$, respectively (Table I and Fig. 8). They agree well with those given by present TB Hamiltonian for nanoclusters. This agreement shows that our parametrization scheme of the orbital shift is more reasonable than that used by Alonso and co-workers, ${ }^{13-15}$ which required very different shifts for different orbits on even the same site.

\section{B. Coordination model and interpolation}

As shown above, local spin and orbital magnetic moments in larger clusters depend mainly on local coordination num- 


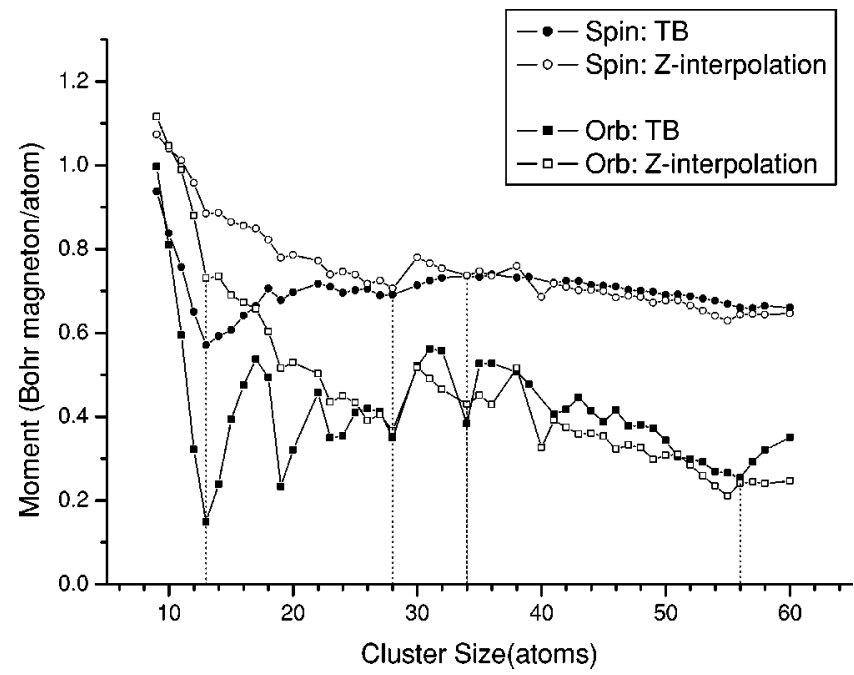

FIG. 9. Comparison between the calculated spin and orbital moments by the $Z$-interpolation model and TB calculations. Vertical dashed lines show theoretically predicted positions of moment minima.

bers, being less sensitive to the cluster size. Therefore, we expect that the $Z$-interpolation model, Eq. (11), can describe the spin and orbital moment variations approximately. As an extension of Jensen and Bennemann's coordination model, ${ }^{7}$ we assume that both local spin and orbital moments are determined by their coordination numbers, with values taken from Eq. (11). Neglecting the noncollinearity, the total moment of a cluster is obtained by

$$
\mu=\mu_{\text {spin }}+\mu_{\text {orb }}=\frac{1}{n} \sum_{i} \mu_{\text {orb }}\left(Z_{i}\right)+\frac{1}{n} \sum_{i} \mu_{\text {spin }}\left(Z_{i}\right) .
$$

For clusters of the MIAL structure, $\mu_{\text {spin }}$ and $\mu_{\text {orb }}$ obtained from this $Z$-interpolation are shown in Fig. 9, together with those calculated from the self-consistent TB model (already shown in Fig. 4) for comparison.

For $\mathrm{Ni}_{n}$ with $n \geqslant 22$, the magnetic moments obtained from this Z-interpolation agree well with those from our selfconsistent TB calculations. Their differences are less than $\pm 0.05 \mu_{B}$ /atom for spin moments, and less than \pm 0.1 $\mu_{B} /$ atom for orbital ones. This shows that the surface enhancement effect given by Hamiltonian (1) could be reproduced approximately by considering only the coordination dependence.

Since this scheme is so simple and elegant, it is of interest to see to what extent it could be applied. Although there are experimental measurements on magnetic moments for clusters with $n>100$, no experimental and theoretical studies on geometrical structures are available. We thus only consider nearly spherical clusters cut ideally from a fcc crystal. In order to ensure these structures to have the least relaxation on cluster surfaces which could be meaningfully compared with experiments, all clusters chosen to plot in Fig. 10 by the $Z$-interpolation possess atoms with coordination larger than 6. The experimental results of Refs. 2 and 3 are shown in

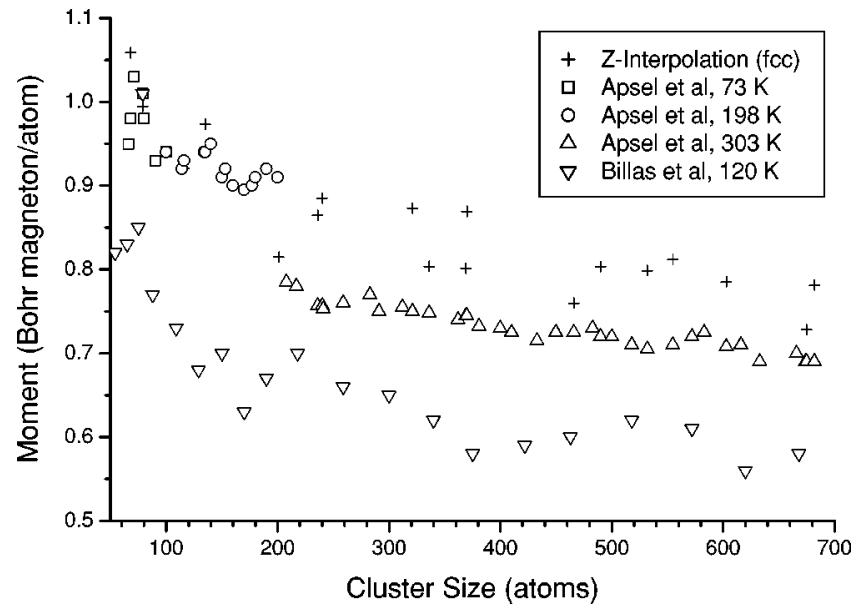

FIG. 10. Total moment of clusters up to $n=700$, obtained by the $Z$-interpolation model (crosses) and experiments (open symbols).

Fig. 10 as comparison. The Z-interpolation results are in good agreements with those of Apsel et al. for clusters with $n<200$. When $n>200$, the experimental moments are about $0.1 \mu_{B}$ /atom lower than the $Z$-interpolation values. This difference might be due to the temperature effect, because in this range of cluster size, Apsel et al. made their measurement at an elevated temperature $T=303 \mathrm{~K}$. As shown in their paper (Fig. 3 of Ref. 3), this elevation of measuring temperature may indeed cause such an amount of moment decrease. However, we cannot offer a reasonable explanation to another earlier experiment by Billas et al. which, though claimed to be performed at temperature $T=120 \mathrm{~K}$, gave much lower magnetic moments (Fig. 10).

We conclude at this point that for large Ni clusters ( $n$ $\geqslant 22$ ), local geometrical structure plays an important role in determining the magnetic moments and a $Z$-interpolation model gives good estimates on the size dependences of magnetic moment. However, one should notice that the experimental moment minima ( $n=13,34$, and 56 , shown by vertical lines in Fig. 9), which are quite prominent in TB calculations and independent of the choice of $U$ parameter, become much less prominent in the $Z$-interpolation results. Thus this $Z$-interpolation scheme is not precise enough to give the delicate features on the size dependence curve. Even more important point to be noticed here is that, comparing with the TB calculation, for the small clusters $(n \leqslant 20)$, as shown in Fig. 9, the moments obtained by considering only surface enhancement are rather large. For example, at $n$ $=13$, we have $\mu_{\text {spin }}=0.88 \mu_{B}$ /atom and $\mu_{\text {orb }}$ $=0.73 \mu_{B}$ /atom according to the $Z$-interpolation, much larger than the corresponding TB values $0.57 \mu_{B}$ /atom and $0.15 \mu_{B}$ /atom. We will discuss the physics behind this huge deviation in the following section.

\section{QUANTUM CONFINEMENT}

Since the moment variations in small clusters cannot be explained by surface enhancements only, we study the densities of states (DOS) in such systems to explore other phys- 


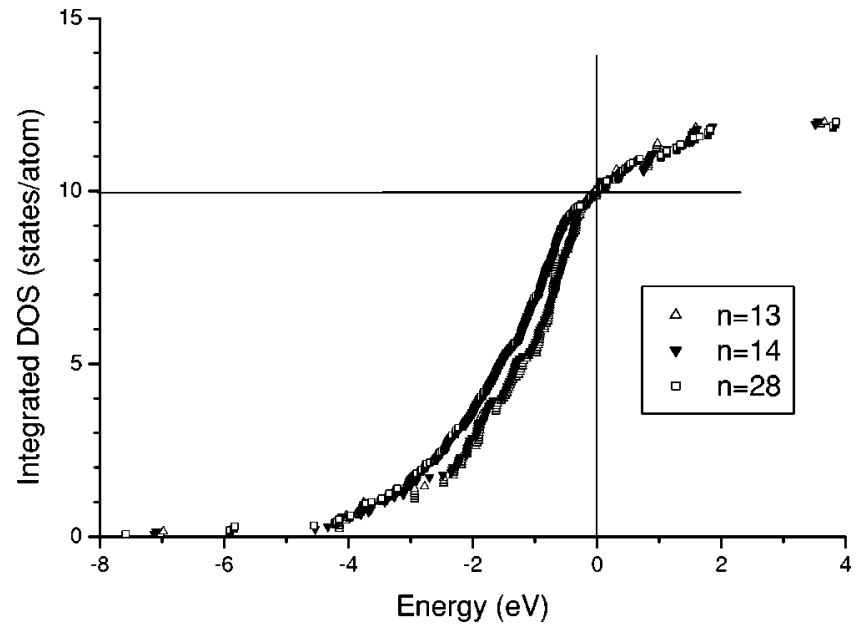

FIG. 11. Integrated density of states per atom for three clusters with $n=13,14$, and 28, with the Fermi level set as zero.

ics. The integrated DOS per atom are shown in Fig. 11 for $\mathrm{Ni}_{13}, \mathrm{Ni}_{14}$, and $\mathrm{Ni}_{28}$. Enlarged plots are shown in Fig. 12(a) for an energy window below the $d$ band bottom and in Fig. 12(b) for another energy window near the Fermi (highest occupied) energy. It is noted that different units of DOS are used in Figs. 11 and 12. It is obvious from Fig. 11 that the electron occupation is dominated by the $d$ bands, spreading from -4 to $2 \mathrm{eV}$ with respect to the Fermi energy. Due to the very localized nature of $d$ orbits, DOS shown in Fig. 11 are very similar for all three clusters. However, below the $d$ band bottom as shown in Fig. 12(a), very characteristic features due to quantum confinement appear. The first two states, one spin up and another spin down, consist of the atomic $s$ orbits of all atoms of clusters $n=13,14$, or 28 . These two states show a global S symmetry, marked as $1 S$ in Fig. 12(a). The next six states [marked 1P in Fig. 12(a)] consist also of the atomic $s$ orbits of all atoms inside a cluster, but have global $P$ symmetry over the whole cluster. Energies of these eight states are lower than the $d$ band minimum, and are all occupied for $n=13,14$, and 28. Their energies decrease with increasing the cluster size, and the degeneracy of 1P states depends on the shape of clusters$1 P$ states are perfectly degenerate for nearly spherical clusters with $n=13$ and 28, but are splitted for the prolate cluster $\mathrm{Ni}_{14}$.

At a higher energy region, we find other ten states to consist mainly of atomic $s$ orbits of all atoms but show a global $D$ symmetry. These states are marked as $1 D$ in Fig. 12(b), with five states spin up and others spin down. For $\mathrm{Ni}_{13}$, these ten states are almost degenerate. The small energy differences are induced by very small exchange interactions, because these states contain mainly atomic $s$ orbits and only small $d$ components through hybridization. For $\mathrm{Ni}_{13}$, all ten $D$ states are about $1 \mathrm{eV}$ above the Fermi energy. For $\mathrm{Ni}_{14}$, the structure is prolonged along one direction and the symmetry is lowered. As a consequence, the energies of two states, marked as $1 D(\sigma)$ in Fig. 12(b), are greatly lowered and become occupied; while the remaining eight states,
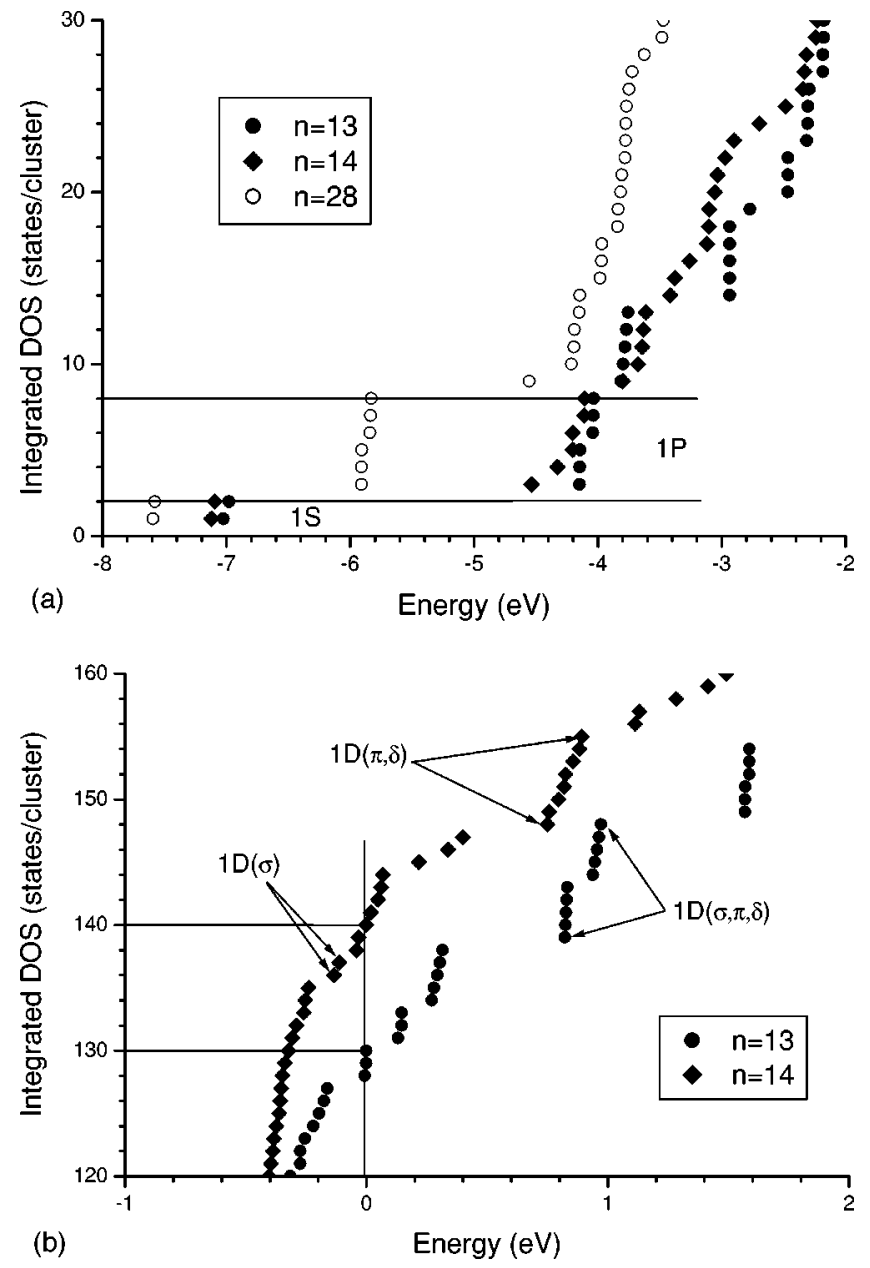

FIG. 12. Integrated density of states per cluster in an energy window [ $-8 \mathrm{eV},-2 \mathrm{eV}]$ for clusters with $n=13,14$, and 28, and (b) in another energy window $[-1 \mathrm{eV}, 2 \mathrm{eV}]$ for clusters with $n$ $=13,14$. Quantumly confined states are denoted in the figures by their global symmetries.

marked as $1 D(\pi, \delta)$ in Fig. 12(b), are still left empty above the Fermi energy.

This volume confinement leads to quantum terraces in the $s$ electron occupation against the cluster size as shown in Fig. 13. With large enough size, we find the $s$-electron occupation to increase almost linearly, about $0.72 s$ electrons per atom as shown by the dashed line in Fig. 13. However, one terrace appears from $n=9-13$ where the total number of $s$ electrons remains eight independent of the cluster size, as shown by the horizontal solid line in Fig. 13. In fact, when the size increase from $n=9-13$, all eight $1 S$ and $1 P$ states are occupied, but all $1 D$ states are empty due to the volume confinement.

The volume confinement on $s$ electrons has great impact on the $d$ band filling. As the total number of electrons increases from $n=9$ to 13 , since the $s$ derived global $S$ and $P$ states can hold only eight electrons, the additional electrons have to fill the $d$ derived bands. This is shown by the sharp increase of the average $d$-electron occupation from $n=9$ to 13 in Fig. 14. The $d$-electron occupation reaches a maximum 


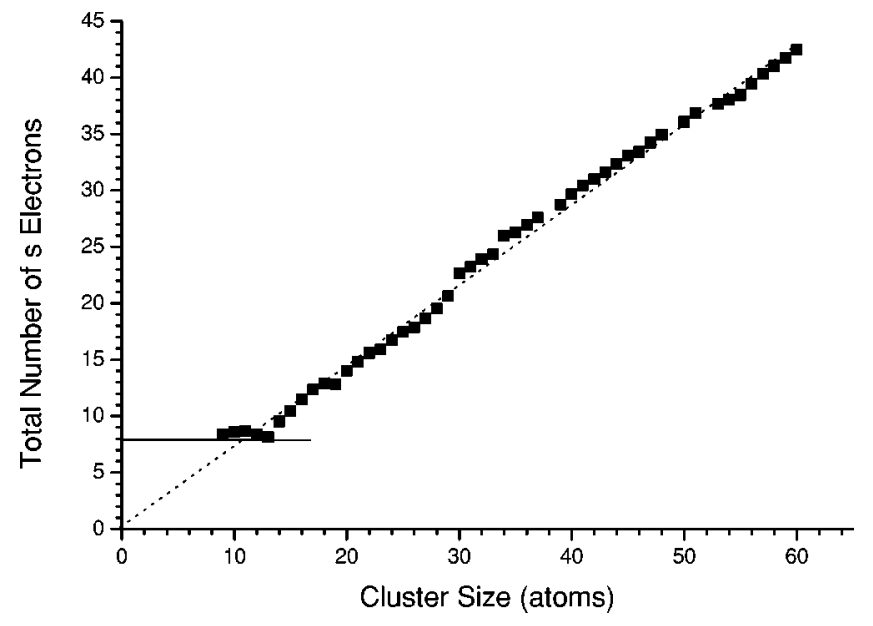

FIG. 13. Total number of $s$ electrons as a function of cluster size. Dotted line shows the average number of $s$ electrons per atom. Terrace (horizontal solid line) at total $s$ electron number 8 from $n$ $=9$ to 13 shows the quantum effect of volume confinement.

at $\mathrm{Ni}_{13}$. Assuming full occupancy of the spin up $d$ bands, the number of spin-down holes could be simply estimated by the quantum confined number of $s$ electrons, giving 8/9=0.889 and $8 / 13=0.615$ holes per atom, for $\mathrm{Ni}_{9}$ and $\mathrm{Ni}_{13}$, respectively. This is just the spin moments obtained by all firstprinciples LSDA calculations (Reddy et al. and Reuse et al. in Fig. 3) and approximately by TB calculations too (present and Guevara et al. in Fig. 3) except that of Alsonso et al. This explains why the spin moment of $\mathrm{Ni}_{13}$ shows such a sharp minimum, and the total moment of $\mathrm{Ni}_{13}$ drops even below that of many larger clusters in experiments.

The average $d$-electron occupation is about 9.04 for clusters with $n>30$ and is about 9.08 for clusters with $17<n$ $<28$. Compared with Fig. 2, the step at 28 is probably related to the structure transition. With increasing the cluster size, the quantum confinement effect becomes small. Taking the difference between TB and Z-interpolation result (Fig. 9)

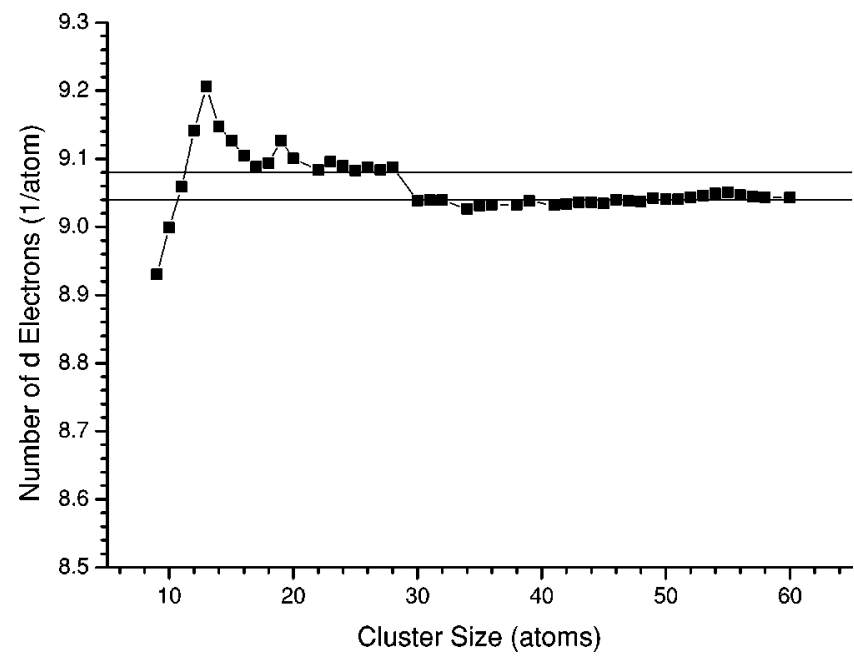

FIG. 14. Number of average $d$ electrons per atom as a function of cluster size. The peak at $n=13$ explains the deep minimum of both spin and orbital moment at this cluster size. as a measure to describe this quantum confinement, we note again that the effect on orbital moment is roughly twice as that on spin moment.

Fujima and Yamaguchi ${ }^{8}$ also considered this quantum confinement effect, and assigned speculatively the oscillations of magnetic moment to the quantum filling of a series of $s$-derived states. Our calculations confirm that the minimum at $\mathrm{Ni}_{13}$ is mostly due to this origin, but other oscillations at larger size are mostly from the surface enhancements of orbital moments. Another point, different from their speculations, is that even just above $\mathrm{Ni}_{13}$, the filling of $s$-derived global $D$ states does not happen abruptly because the global $D$ states are no longer degenerate when a cluster becomes less symmetric when $n>13$. Instead, the ten global $D$ states are lowered below the Fermi energy gradually, leading to a gradual increase of spin moment when $n$ increases from $n=14$ to about 20 .

\section{SUMMARY AND CONCLUSIONS}

In addition to the hopping, exchange, and SOC terms which are usually used in previous TB calculations, we show that orbital correlation and valence orbital shift due to the electron spillover from the surface atoms to the vacuum are important in the study of nanocluster magnetism. They have been included properly in the TB Hamiltonian used in this paper. With this rather general and unified formalism, we are able to show, in a consistent way, that both spin and orbital magnetic moments of Ni clusters are sensitive to the coordination numbers, and are influenced by quantum confinement in very small clusters.

The calculated magnetic moments of $\mathrm{Ni}_{n}$ clusters with $n$ between 9 and 60, are in better agreements with the experimental results than previous theoretical treatments. For atomic sites with $Z \geqslant 8$ (i.e., bulk and strongly bonding surface atoms), both $\mu_{\text {orb }}$ and $\mu_{\text {spin }}$ are only slightly enhanced compared with bulk values, but the enhancement is great for those with $Z \leqslant 6$ (i.e., weakly bonding surface atoms). A simple coordination $Z$ interpolation is found to account approximately for the size dependence of magnetic moment when $n \geqslant 22$. Thus, the coordination number (i.e., geometrical structure) plays a main role in determining the magnetic moments of large Ni clusters, and a proper TB calculation is necessary to give precisely the moment values and exact positions of moment minima. Identification of minima is made possible by analyzing our TB calculation results. Sharp minimum at $\mathrm{Ni}_{13}$ is contributed by both orbital and spin moments, and due to both quantum confinement and surface enhancement effect. Minima at $\mathrm{Ni}_{34}$ and $\mathrm{Ni}_{56}$ are contributed mostly by orbital moments, and mainly due to surface enhancement effect.

Quantum confinement effect is the main reason that magnetic moment of $\mathrm{Ni}_{13}$ reaches such a pronounced sharp minimum. In small clusters $(n \leqslant 20)$, this effect has strong influences on the $d$ hole number, and $\mu_{\text {spin }}$ and $\mu_{\text {orb }}$ are greatly decreased due to the decrease of $d$ holes. The change in $\mu_{o r b}$ due to decreasing $d$ holes is about twice as large as in $\mu_{\text {spin }}$ for $\mathrm{Ni}$ atoms, as expected from Hund's rules for the case when $d$ hole is less than 1 . As a result, orbital magnetic 
moment plays the most important role in the moment oscillations versus cluster size.

Despite of the good agreements achieved, there are still some discrepancies between our results and the experimental ones. For example, a moment minimum at $n=28$ appears in our theoretical calculations, but it does not appear in experiments. This may be attributed to the TIC to MIC structure transition at $n=27$ and 28. Also, it is shown that for many other $\mathrm{Ni}$ clusters, energy differences between the ground state and its closest isomer might be very small. ${ }^{31}$ These might lead to discrepancies between experiments and present theory, which is a subject of further investigations.

\section{ACKNOWLEDGMENTS}

X.G.W. and D.S.W. acknowledge the visiting scholar program of National Center of Theoretical Sciences, Hsinchu, Taiwan, which initiated this collaborative research. D.S.W. also acknowledges support by Grants No. 10234010, of National Science Foundation of China, and No. G19990328-02, of National Pan-deng Project of China. J.M.D. and X.G.W. acknowledge support by Grant No. 10074026 and Grant No. 10304007, of National Science Foundation of China, and a grant for the State Key Program for Basic Research of China.
${ }^{1}$ I.M.L. Billas, J.A. Becker, A. Châtelain, and W.A. de Heer, Phys. Rev. Lett. 71, 4067 (1993); Science 265, 1682 (1994).

${ }^{2}$ I.M.L. Billas, A. Châtelain, and W.A. de Heer, J. Magn. Magn. Mater. 168, 64 (1997).

${ }^{3}$ S.E. Apsel, J.W. Emmert, J. Deng, and L.A. Bloomfield, Phys. Rev. Lett. 76, 1441 (1996).

${ }^{4}$ M.B. Knickelbein, J. Chem. Phys. 116, 9703 (2002).

${ }^{5}$ A.J. Freeman and R.Q. Wu, J. Magn. Magn. Mater. 100, 497 (1991)

${ }^{6}$ E. Wimmer, H. Krakauer, M. Weinert, and A.J. Freeman, Phys. Rev. B 24, 864 (1981); M. Weinert, E. Wimmer, and A.J. Freeman, ibid. 26, 471 (1982).

${ }^{7}$ P.J. Jensen and K.H. Bennemann, Z. Phys. D: At., Mol. Clusters 35, 273 (1995).

${ }^{8}$ N. Fujima and T. Yamaguchi, Phys. Rev. B 54, 26 (1996).

${ }^{9}$ F.A. Reuse, S.N. Khanna, and S. Bernel, Phys. Rev. B 52, R11650 (1995); F.A. Reuse and S.N. Khanna, Chem. Phys. Lett. 234, 77 (1995).

${ }^{10}$ B.V. Reddy, S.K. Nayak, S.N. Khanna, B.K. Rao, and P. Jena, J. Phys. Chem. A 102, 1748 (1998); S.K. Nayak, S.N. Khanna, B.K. Rao, and P. Jena, ibid. 101, 1072 (1997).

${ }^{11}$ N. Fujima and T. Yamaguchi, Mater. Sci. Eng., A 217, 295 (1996).

${ }^{12}$ J. Guevara, F. Parisi, A.M. Llois, and M. Weissmann, Phys. Rev. B 55, 13283 (1997); G. Fabricius, A.M. Llois, M. Weissmann, and M.A. Khan, ibid. 49, 2121 (1994).

${ }^{13}$ J.A. Alonso, Chem. Rev. (Washington, D.C.) 100, 637 (2000).

${ }^{14}$ S. Bouarab, A. Vega, M.J. López, M.P. Iñiguez, and J.A. Alonso, Phys. Rev. B 55, 13279 (1997); F. Aguilera-Granja, S. Bouarab, M.J. López, A. Vega, J.M. Montejano-Carrizales, M.P. Iniguez, and J.A. Alonso, ibid. 57, 12469 (1998).

${ }^{15}$ J.L. Rodríguez-López, F. Aguilera-Granja, A. Vega, and J.A. Alonso, Eur. Phys. J. D 6, 235 (1999).
${ }^{16}$ L. Zhou, D.S. Wang, and Y. Kawazoe, Phys. Rev. B 60, 9545 (1999).

${ }^{17}$ M. Komelj, C. Ederer, J.W. Davenport, and M. Fähnle, Phys. Rev. B 66, 140407 (2002).

${ }^{18}$ B. Nonas, I. Cabria, R. Zeller, P.H. Dederichs, T. Huhne, and H. Ebert, Phys. Rev. Lett. 86, 2146 (2001).

${ }^{19}$ R.A. Guirado-López, J. Dorantes-Dávila, and G.M. Pastor, Phys. Rev. Lett. 90, 226402 (2003).

${ }^{20}$ M.J. López and J. Jellink, Phys. Rev. A 50, 1445 (1994).

${ }^{21}$ R.P. Gupta, Phys. Rev. B 23, 6265 (1981); F. Ducastelle, J. Phys. (Paris) 31, 1055 (1970).

${ }^{22}$ J.M. Montejano-Carrizales, M.P. Iniguez, J.A. Alonso, and M.J. López, Phys. Rev. B 54, 5961 (1996).

${ }^{23}$ We acknowledge M. J. López and her colleagues for kindly providing us the Cartisian coordinates of MIAL structure of $\mathrm{Ni}_{n}$ clusters of $n=1-60$ in private communication.

${ }^{24}$ D. A. Papaconstantopoulos, Handbook of the Band Structure of Elemental Solids (Plenum Press, New York, 1986).

${ }^{25}$ V. Heine, Phys. Rev. 153, 673 (1967); O.K. Andersen and O. Jepsen, Phys. Rev. Lett. 53, 2571 (1984).

${ }^{26} \mathrm{P}$. Bruno, Magnetismus von Festkörpern und Grenzflächen (KFA, Jülich, 1993), Chap. 24.

${ }^{27}$ L. Zhou, J.T. Wang, D.S. Wang, and Y. Kawazoe, Mater. Trans., JIM 40, 1237 (1999).

${ }^{28}$ A.I. Liechtenstein, V.I. Anisimov, and J. Zaanen, Phys. Rev. B 52, R5467 (1995).

${ }^{29}$ V.I. Anisimov, J. Zaanen, and O.K. Andersen, Phys. Rev. B 44, 943 (1991).

${ }^{30}$ B.T. Thole, P. Carra, F. Sette, and G. van der Laan, Phys. Rev. Lett. 68, 1943 (1992).

${ }^{31}$ Y. Xiang, D.Y. Sun, and X.G. Gong, J. Phys. Chem. A 104, 2746 (2000). 